\title{
Intra- and Inter-Rater Reliability of Thickness Measurement of the Psoas Major Muscle Using the Spring Gauge Technique for the Consistent Inward Pressures on Healthy Subjects: An Ultrasonographic Study
}

\author{
In-cheol Jeon ${ }^{1}$, BHSc, PT, Yong-wook $\mathrm{Kim}^{2}$, PhD, PT, Sung-dae Choung ${ }^{1}$, BHSc, PT \\ ${ }^{1}$ Dept. of Physical Therapy, The Graduate School, Yonsei University \\ ${ }^{2}$ Dept. of Physical Therapy, College of Medial Sciences, Jeonju University
}

\begin{abstract}
The purpose of the current study was to determine the intra- and inter-rater reliability of muscle thickness (MT) measurement of the psoas major (PM) using ultrasonography (US) conducted at different inward pressures of approximately $.5 \mathrm{~kg}, 1.0 \mathrm{~kg}, 1.5 \mathrm{~kg}$, and $2.0 \mathrm{~kg}$. Twelve healthy male subjects were recruited for the study. The thicknesses of both PMs of each subject were measured by two different examiners in a random manner to assess the intra- and inter-rater reliability. The measurement values were analyzed using the intra-class correlation coefficient (ICC) with a $95 \%$ confidence interval (CI). ICC $(2,1)$ was used to determine the inter-rater reliability and ICC $(3,1)$ was used to assess the intra-rater reliability of the MT measurement of the PM. The results indicated higher ICC values for intra-rater reliability compared to inter-rater reliability. In addition, the value for intra-rater reliability with $.5 \mathrm{~kg}$ inward pressure $[\mathrm{ICC}=.99(95 \% \mathrm{CI}=.98 \sim .99)]$ was higher compared to $1.0 \mathrm{~kg}, 1.5 \mathrm{~kg}$, and $2.0 \mathrm{~kg}$. Other inward pressures for intra- and inter-rater reliability in current study were also demonstrated to have excellent values ( $\mathrm{ICC}=.94 \sim .99$ ). These findings showed that maintaining consistent inward pressure is essential for maintaining reliability of the results when the MT of the PM is measured by different examiners in a clinical setting.
\end{abstract}

Key Words: Intra-rater reliability; Inter-rater reliability; Inward pressure; Psoas major; Ultrasonography.

\section{Introduction}

The psoas major muscle (PM) plays a role in stabilizing the spine (Barker et al, 2004). Cooper et al (1992) stated that the cross $^{-}$sectional area (CSA) of the ipsilateral PM was significantly decreased in $\mathrm{pa}^{-}$ tients with low back pain (LBP) and disc herniation. Another study stated that PM is considered as a lateral stabilizer of the lumbar spine (Santaguida and McGill, 1995). In addition, the clinical management for improving PM can contribute to the increase of lumbopelvic stability of patients with LBP (Sajko and Stuber, 2009).

Keagy et al (1966) reported that the PM plays an important role in moving the limb forward while walking and acts as a trunk controller from postural deviation while sitting. Another study showed that muscle activation of PM increased during upright standing, forward bending, and lifting (Nachemson, 1966; Nachemson, 1968). These findings support the claim that the PM has an important role with re $^{-}$ spect to lumbar spine stability and movement (Sajko and Stuber, 2009). Since the effective evaluation of the PM is believed to be an important component in rehabilitation in patients with LBP (Sajko and Stuber, 2009), The precise measurement were $\mathrm{re}^{-}$ quired to evaluate the PM in the clinical setting for selective exercise training (Barker et al, 2004).

Corresponding author: Yong-wook Kim ptkim@jj.ac.kr 
Although the isolated force measurement of the PM is not possible because it coordinates with the iliacus, psoas minor, adductor group, and rectus femoris (Sajko and Stuber, 2009), indirect measurement may be provided by the muscle thickness (MT), which indicates the capacity of the muscle force. Therefore, the accurate and meaningful values in the measurement of the MT are important (Rankin et al, 2006; Teyhen et al, 2007).

Ultrasonography (US) of PM is often involved in the measurement of MT as a convenient method for determining the CSA of muscles (Abe et al, 1994; Takai et al, 2011). Force generation is closely related to muscle CSA (Akagi et al, 2009; Jubrias et al, 1997). The MT measurement using US has been closely correlated to muscle CSA (Abe et al, 1997; Sipilä and Suominen, 1991). Measurement using US to investigate the MT has been performed in both clinical and research settings (Akbari et al, 2008). Although the magnetic resonance imaging (MRI) and computed tomography (CT) are widely used to estimate the volume of the PM (McGill et al, 1993; Santaguida and McGill, 1995), US has been increasingly used to measure the MT because of similar advantages to CT or MRI in visualizing muscle tissues (Takai et al, 2011). The use of CT and MRI may be the most accurate way to examine the body tissue. However, the disadvantages of $\mathrm{CT}$ related to radiation exposure are still being actively investigated. In addition, precise measurement techniques for body tissue evaluation such as MRI also have disadvantages because they are time consuming, expensive, and involve a high radiation dose (Snijder et al, 2002).

During the measurement of the MT using US carried out by different examiners, the effort to maintain a consistent position, orientation, and inward pressure of the transducer is required to enhance the reliability of the results (Ishida and Watanabe, 2012; Teyhen et al, 2007). Various studies have reported that high intra- and inter-rater reliability were established for two different examiners who measured stabilizer muscles such as the multifidus and longus colli (Cagnie et al, 2009; Wallwork et al, 2007). However, to the best of our knowledge, no study has evaluated the influence of different inward pressures of the transducer on the PM to measure the MT with by two different examiners using US. Therefore, the aim of our study was to determine the intra- and inter-rater reliability of thickness measurement for the PM using US with the different inward pressure of $.5 \mathrm{~kg}, 1.0 \mathrm{~kg}, 1.5 \mathrm{~kg}$, and $2.0 \mathrm{~kg}$ with two different raters. We hypothesized that maintaining consistent inward pressure would result in excellent values for inter- and intra-rater reliability at all different inward pressures $(.5 \mathrm{~kg}, 1.0 \mathrm{~kg}, 1.5 \mathrm{~kg}$, and $2.0 \mathrm{~kg})$.

\section{Methods}

\section{Subjects}

University students were recruited as subjects for the present study. Twenty healthy male subjects were included; thus, measurements were taken from 40 PMs (Table 1). The exclusion criteria were as follows: 1) metabolic, neuromuscular, or musculoskeletal disorders; 2) LBP; and 3) pain in any region of the body while testing. The primary investigator explained all procedures to the subjects before the start of the measurements and consent was given prior to participation. Before the experiment, the experimental protocols were explained in detail to all of the subjects.

\section{Instrument}

The US imaging was collected by two examiners

Table 1. General characteristics of the subjects

$(\mathrm{N}=40)$

\begin{tabular}{cr}
\hline \hline Variable & Mean \pm SD \\
\hline Age $(\mathrm{yr})$ & $23.4 \pm 2.2$ \\
Height $(\mathrm{cm})$ & $176.7 \pm 3.8$ \\
Weight $(\mathrm{kg})$ & $69.1 \pm 7.1$ \\
\hline
\end{tabular}




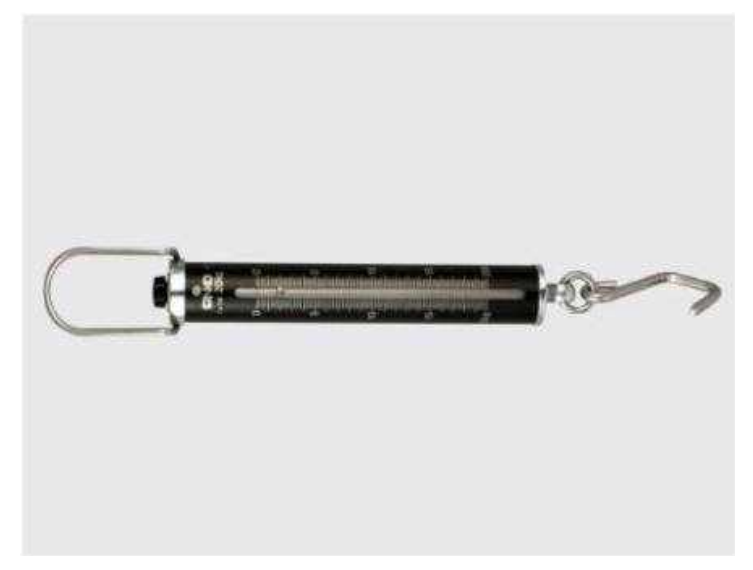

Figure 1. Spring gauge used to measure inward pressure on the psoas major.

who had the experience with MT measuremen $\mathrm{t}$ using US $7.5 \mathrm{MHz}$ linear transducer (SonoAce x8, Medison Co., Ltd., Seoul, Korea) was utilized for US to measure the MT of the PM. In order to ensure that the ultrasound transducer maintained consistent inward pressures of $.5 \mathrm{~kg}, 1.0 \mathrm{~kg}, 1.5 \mathrm{~kg}$, and $2.0 \mathrm{~kg}$, spring gauge (Kirin Co., Ltd., Seoul, Korea) was used; this was suspended from the a perpendicular fixed bar (Figure 1). This measurement application had a force-measuring capacity of 0.0 to $30.0 \mathrm{~kg}$, in $.1 \mathrm{~kg}$ increments. The inward pressures were applied perpendicular to the skin surface on the PM to ensure precise force generation (Hayes et al, 2002). The transducer was secured to end of the spring gauge by using leukotape $\mathrm{P}$ nonelastic sports tape (BSN Medical, Hamburg, Germany) to allow both the fixed bar and the spring gauge.

\section{Procedures}

The subjects were placed in supine position on the rigid surface of a height-adjustable table. The inward pressure set at $0 \mathrm{~kg}$ in contact with the skin during the application of transducer using the height-adjustable table in order to precisely apply the four different inward pressures (Figure 2). The arms of the subjects were crossed over their chests. The four different inward pressures $(.5 \mathrm{~kg}, 1.0 \mathrm{~kg}, 1.5 \mathrm{~kg}$, $2.0 \mathrm{~kg}$ ) were applied using the spring gauge to per-

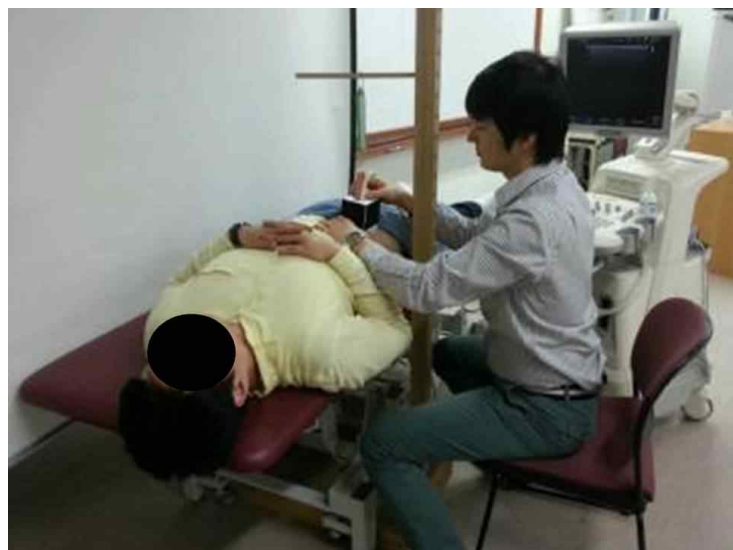

Figure 2. Measurement performed with a handheld ultrasonography transducer and spring gauge.

form consistent screening of the PM. The transducer was placed transversely on both sides of the PM medial to the anterior superior iliac spine and observed above the level of the inguinal ligament (Sajko and Stuber, 2009; Tatu et al, 2001). The images captured by US were saved to calculate the MT of the PM. The angle of the transducer was tilted to position the transducer in a perpendicular plane to the muscle fascia of the PM (Jhu et al, 2010). The location of the transducer was marked with a straight line on the skin to control the specific contact area between the two surfaces of the transducer and the skin. After these procedures, only the difference in inward pressures was re-measured using the same procedure. The MT was calculated using the average values of three measurements for each condition for analysis.

Two experienced examiners [one with five years' experience (examiner 1), the other with two years' experience (examiner 2)] took measurements from both PMs on each subject. All measurements were performed in the same day to assess intra- and inter-rater reliability (Figure 3). The measurement order of examiners and inward pressure were randomized by randomly changing the order of application of the different inward pressures of the transducer and of the measurement performed by the examiners. The examiners were blinded to the results of the 


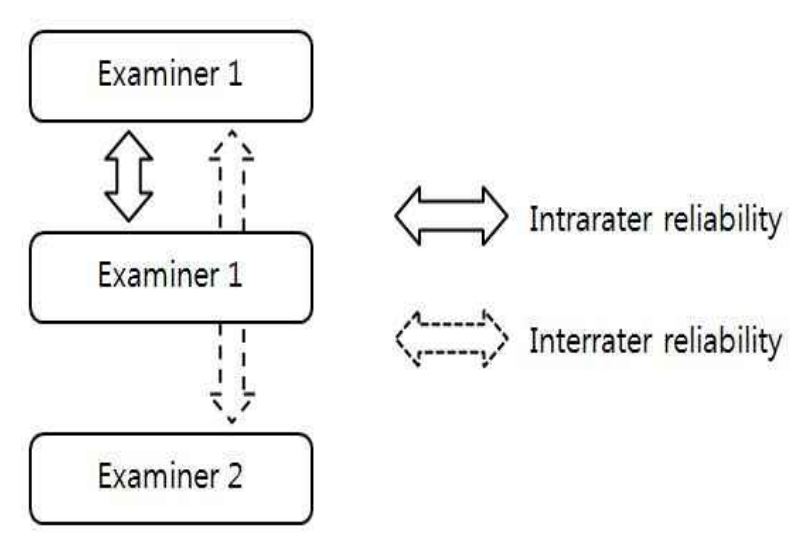

Figure 3. Flow chart of the measurement progression.

measurement and the subject information. The primary investigator was involved in recording all $\mathrm{re}^{-}$ sults from examiners. In order to carry out the experimental procedure, the examiners were familiarized with US and the generation of sensitive force with the spring gauge before performing the actual measurements.

\section{Statistical Analysis}

All statistical analyses were conducted using SPSS ver. 18.0 software (SPSS Inc., Chicago, IL, USA). Intra-class correlation coefficients (ICCs) and 95\% confidence Intervals (CIs) were used. The $\operatorname{ICC}(3,1)$ model to estimate the intra-rater reliability and the ICC $(2,1)$ model was used to test the inter-rater reliability. We used averaged data from three $\mathrm{re}^{-}$ peated measurements of the same image to $\mathrm{de}^{-}$ termine the MT in each condition. Mean measurement thickness with standard deviations (SDs) of the three measurements were calculated for each session. Minimal detectable difference (MDD) was calculated using the following formula: [MDD95=1.96 $\times \mathrm{SEM} \times$ $\sqrt{2}$ ]. The aim of this was to determine the magnitude of change that would exceed the minimal error of the measurement at a $95 \% \mathrm{CI}$. One-way repeated analysis of variance was used to determine differences between PM thickness according to the different inward pressures $(.5 \mathrm{~kg}, 1.0 \mathrm{~kg}, 1.5 \mathrm{~kg}$ and 2.0 $\mathrm{kg}$ ) of the transducer. The Bonferroni test was performed for post hoc analysis. Values were considered statistically significant at $a<.05$.

\section{Results}

The ICC for intra-rater reliability was higher than or at least equal to .97 , which is an excellent result. In addition, the ICC for intra-rater reliability was higher than inter-rater reliability in all of the different inward pressures. The MT of the PM decreased in the order of inward pressure conditions of $.5 \mathrm{~kg}$, $1.0 \mathrm{~kg}, 1.5 \mathrm{~kg}$ and $2.0 \mathrm{~kg}(\mathrm{p}<.05)$. The values for intra- and inter-rater reliability with $.5 \mathrm{~kg}, 1.0 \mathrm{~kg}, 1.5$ $\mathrm{kg}$, and $2.0 \mathrm{~kg}$ are shown (Table 2) (Table 3). Lesser values of the coefficient of variance $(\mathrm{CV})$ and the standard error of measurement (SEM) of intra-rater reliability were shown compared with inter-rater reliability (Table 2) (Table 3). In addition, the values

Table 2. Intra-rater reliability of the measurement of the psoas major using ultrasonography with different inward pressures

\begin{tabular}{ccccc}
\hline \hline \multirow{2}{*}{$\mathrm{PM}^{\mathrm{a}}$} & \multicolumn{4}{c}{ Intra-rater reliability } \\
\cline { 2 - 5 } & $\mathrm{ICC}^{\mathrm{b}}\left(95 \% \mathrm{CI}^{\mathrm{c}}\right)$ & $\mathrm{SEM}^{\mathrm{d}}(\mathrm{mm})$ & $\mathrm{MDC}^{\mathrm{e}}(\mathrm{mm})$ & $\mathrm{CV}^{\mathrm{f}}(\%)$ \\
\hline $.5 \mathrm{~kg}$ & $.99(.98 \sim .99)$ & .04 & .10 & 27 \\
$1.0 \mathrm{~kg}$ & $.97(.95 \sim .99)$ & .07 & .19 & 32 \\
$1.5 \mathrm{~kg}$ & $.98(.95 \sim .99)$ & .05 & .15 & 36 \\
$2.0 \mathrm{~kg}$ & $.98(.97 \sim .99)$ & .05 & .14 & 37 \\
\hline
\end{tabular}

apsoas major, ${ }^{\mathrm{b}}$ intra-class correlation coefficients, ${ }^{\mathrm{c}}$ confidence interval, ${ }^{\mathrm{d}}$ standard error of measurement, ${ }^{\mathrm{e}}$ minimal detectable change, ${ }^{\mathrm{f}}$ coefficient of variance, $\mathrm{p}<.05$. 
for ICC with 95\% CI of intra- and inter-rater reliability, including $\mathrm{MDC}$, are presented (Table 2)(Table 3). In the interpretation, the ICC values $>.75$ were considered to indicate excellent reliability; .40 .75 indicated fair to good reliability; and $.00 \sim .40$ indicates poor reliability (Crossley et al, 2004). A summary description of thickness changes in PM measurements is provided concerning intra-rater reliability in Table 4. The difference of the mean values on MT of PM between the inward pressures was descibed in Table 5.

\section{Discussion}

This study investigated the changes in the MT of the PM induced by different inward pressures of approximately $.5 \mathrm{~kg}, 1.0 \mathrm{~kg}, 1.5 \mathrm{~kg}$, and $2.0 \mathrm{~kg}$ to $\mathrm{de}^{-}$ termine the intra- and inter-rater reliability of the measurements using the US imaging technique. We believe that the current study is the first reported study to determine the intra- and inter-rater reliability of US for the measurement of the MT of the PM.

The high reliability in the current study was influenced by the experimental protocol used to ensure standardization of the measurement using various components. In this study, a height-adjustable table was used to adjust the precise height for the transducer in contact with the skin surface on the PM in response to the different inward pressures. Furthermore, the contact moment with consistent pressure could be obtained through height adjustment between the transducer and the skin surface on the PM. Moreover, the surface of the table was composed of rigid wood boards in contact with the lower limbs of subjects to minimize slight variations of body position because of the downward force of the transducer on the skin surface of the PM. The spring gauge was also used to provide consistent inward pressure of the transducer while measuring the thickness of the PM in response to the different pressures. In addition, the spring gauge was replaced with other spring gauges 4 times after measurements of 5 subjects to avoid loss of the elasticity of springs because of fatigue with extensive use of the spring gauges (Bohannon and Andrews, 1989).

The specific protocols for the measurements in this study might have contributed to the high intra-rater (ICC=.98 .99) and inter-rater reliability (ICC=.94 .97). The current study indicated that the ICC values for intra-rater and inter-rater reliability with $.5 \mathrm{~kg}, 1.0 \mathrm{~kg}, 1.5 \mathrm{~kg}$, and $2.0 \mathrm{~kg}$ inward pressures indicated excellent reliability, thereby support-

Table 3. Inter-rater reliability of the measurement of the psoas major using ultrasonography with difference inward pressure

\begin{tabular}{ccccc}
\hline \hline \multirow{2}{*}{$\mathrm{PM}^{\mathrm{a}}$} & \multicolumn{4}{c}{ Inter-rater reliability } \\
\cline { 2 - 5 } & $\mathrm{ICC}^{\mathrm{b}}\left(95 \% \mathrm{CI}^{\mathrm{c}}\right)$ & $\mathrm{SEM}^{\mathrm{d}}(\mathrm{mm})$ & $\mathrm{MDC}^{\mathrm{e}}(\mathrm{mm})$ & $\mathrm{CV}^{\mathrm{f}}(\%)$ \\
\hline $.5 \mathrm{~kg}$ & $.97(.95 \sim .98)$ & .06 & .18 & 27 \\
$1.0 \mathrm{~kg}$ & $.94(.87 \sim .98)$ & .10 & .27 & 34 \\
$1.5 \mathrm{~kg}$ & $.94(.84 \sim .98)$ & .09 & .26 & 36 \\
$2.0 \mathrm{~kg}$ & $.95(.93 \sim .97)$ & .09 & .24 & 39 \\
\hline
\end{tabular}

apsoas major, ${ }^{\mathrm{b}}$ intra-class correlation coefficients, ${ }^{\mathrm{c}}$ confidence interval, ${ }^{\mathrm{d}}$ standard error of measurement, ${ }^{\mathrm{e}}$ minimal detectable change, ${ }^{\mathrm{f}}$ coefficient of varience, $\mathrm{p}<.05$.

Table 4. Thickness changes of the psoas major muscle

(Unit: mm)

\begin{tabular}{cccccc}
\hline \hline & $.5(\mathrm{~kg})$ & $1.0(\mathrm{~kg})$ & $1.5(\mathrm{~kg})$ & $2.0(\mathrm{~kg})$ & $\mathrm{p}$ value \\
\hline $\mathrm{MT}^{\mathrm{a}}$ of $\mathrm{PM}^{\mathrm{b}}$ & $1.36 \pm .36$ & $1.18 \pm .37$ & $1.02 \pm .36$ & $.89 \pm .33$ & $<.001$ \\
\hline
\end{tabular}

amuscle thickness, ${ }^{b}$ psoas major. 
Table 5. Significant differences between mean values using the Bonferroni test

\begin{tabular}{|c|c|c|c|}
\hline & & \multicolumn{2}{|c|}{ 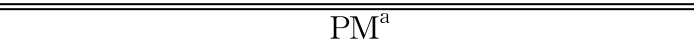 } \\
\hline & & Difference & $\mathrm{p}$ value \\
\hline \multirow[t]{3}{*}{$.5 \mathrm{~kg}$} & versus $1.0 \mathrm{~kg}$ & .180 & $<.001$ \\
\hline & versus $1.5 \mathrm{~kg}$ & .342 & $<.001$ \\
\hline & versus $2.0 \mathrm{~kg}$ & .471 & $<.001$ \\
\hline \multirow[t]{3}{*}{$1.0 \mathrm{~kg}$} & versus $.5 \mathrm{~kg}$ & -.180 & $<.001$ \\
\hline & versus $1.5 \mathrm{~kg}$ & .162 & $<.001$ \\
\hline & versus $2.0 \mathrm{~kg}$ & .291 & $<.001$ \\
\hline \multirow[t]{3}{*}{$1.5 \mathrm{~kg}$} & versus $.5 \mathrm{~kg}$ & -.342 & $<.001$ \\
\hline & versus $1.0 \mathrm{~kg}$ & -.162 & $<.001$ \\
\hline & versus $2.0 \mathrm{~kg}$ & .129 & $<.001$ \\
\hline \multirow[t]{3}{*}{$2.0 \mathrm{~kg}$} & versus $.5 \mathrm{~kg}$ & -.471 & $<.001$ \\
\hline & versus $1.0 \mathrm{~kg}$ & -.291 & $<.001$ \\
\hline & versus $1.5 \mathrm{~kg}$ & -.129 & $<.001$ \\
\hline
\end{tabular}

apsoas major

ing our hypothesis. Previous studies have shown that the inward pressure of the transducer was carried out below $6.0 \mathrm{~N}$ (approximate $.6 \mathrm{~kg}$ ) while measuring abdominal muscles (Ando et al, 2010; Ishida and Watanabe, 2012). Moreover, although the ICC values for internal oblique (IO) and external oblique (EO) muscles exhibited high reliability with approximately $4.0 \mathrm{~N}$ in a previous study, which was close to the inward pressure of $.5 \mathrm{~kg}$ used in current study (Ishida and Watanabe, 2012), intra- and inter-rater reliability were shown even with $1.0 \mathrm{~kg}, 1.5 \mathrm{~kg}$, and $2.0 \mathrm{~kg}$ inward pressures because of the attempt to maintain the consistent inward pressures using the various components described.

In this study, the MT of the PM was slightly decreased according to the different inward pressures of the transducer $(\mathrm{p}<.05)$, although the inward pressures on the skin surface of the PM exhibited subtle changes. The precise measurement of the MT could be affected by the elasticity of the muscles (Ishida and Watanabe, 2012). Thus, the effort to maintain consistent inward pressure using US can be an important factor while measuring $\mathrm{MT}$ in not only the PM, but also all other muscles. A small difference in the MT may be misestimated under inconsistent in- ward pressures of the transducer because of slight MT changes (Akbari et al, 2008; Ishida and Watanabe, 2012). For this reason, the MT measured using different inward pressures can be positively influenced with equipment such as a high-density foam cube (Bunce et al, 2002) and a transducer holder (Ishida and Watanabe, 2012) to promote consistent inward pressures.

A previous study reported hands-free transducer holders can be used practically in both clinical and research settings. However, examination using a US transducer can be performed through the handheld technique in a general clinical setting. Thus, the spring gauge described in this study could be used in the clinical setting because it has similar advantages to the equipment currently used to measure the MT with consistent inward pressure (Bunce et al, 2002; Ishida and Watanabe, 2012). Furthermore, the spring gauge in the current study can be used with single-handed to simultaneously determine the pressure while suspending from the stationary objects; moreover, an additional instrument such as a force plate is not needed for pressure confirmation. In addition, the equipment has advantages such as easy application and cost-effectiveness. These fea- 
tures make it more accessible in the clinical setting when it comes to minimizing possible measurement error due to inconsistent inward pressures of the transducer while measuring the MT.

The current study has several limitations. First, the subjects in this investigation were all male and belonged to a relatively young age group. No clinical setting can be reflected with this procedure, as both male and female patients need to be measured. Second, the results of the current study cannot be generalized to the patient group. Further study to establish the reliability of MT measurements on a male and female patient population is required.

\section{Conclusion}

This research showed that intra- ${ }^{-}$and inter-rater reliability were excellent when maintaining a consistent inward pressures while the MT of the PM was measured by the same and different examiners. Based on the findings of the current study, we recommended that MT could be provided for the PM with reliable results when an effort to maintain consistent inward pressures was considered in the clinical setting. This measurement of the MT of the PM may be useful in ultrasonographic practice and $\mathrm{re}^{-}$ search, as the results in this study implied that inconsistent inward pressures can have a negative effect on the precise measurement on the MT of the PM.

\section{References}

Abe T, Kawakami Y, Suzuki Y, et al. Effects of 20 days bed rest on muscle morphology. J Gravit Physiol. 1997;4(1):S10-S14.

Abe T, Kondo M, Kawakami Y, et al. Prediction equations for body composition of Japanese adults by b-mode ultrasound. Am J Hum Biol. 1994;6(2):161-170.
Akagi R, Takai Y, Ohta M, et al. Muscle volume compared to cross-sectional area is more appropriate for evaluating muscle strength in young and elderly individuals. Age Ageing. 2009;38(5): $564-569$.

Akbari A, Khorashadizadeh S, Abdi G. The effect of motor control exercise versus general exercise on lumbar local stabilizing muscles thickness: Randomized controlled trial of patients with chronic low back pain. J Back Musculoskelet Rehabil. 2008;21(2):105-112.

Ando K, Saito S, Aoki Y, et al. Development of probe scan mechanism for echography using pneumatic actuators and its estimation control in contact force on body surface. J Robot Soc Jpn. 2010;28(7):792-801.

Barker KL, Shamley DR, Jackson D. Changes in the cross-sectional area of multifidus and psoas in patients with unilateral back pain: The relationship to pain and disability. Spine (Phila $\mathrm{Pa}$ 1976). 2004;15;29(22):E515-E519.

Bohannon RW, Andrews AW. Accuracy of spring and strain gauge hand-held dynamometers. J Orthop Sports Phys Ther. 1989;10(8):323-325.

Bunce SM, Moore AP, Hough AD. M-mode ultrasound: A reliable measure of transversus abdominis thickness? Clin Biomech (Bristol, Avon). 2002;17(4):315-317.

Cagnie B, Derese E, Vandamme L, et al. Validity and reliability of ultrasonography for the longus colli in asymptomatic subjects. Man Ther. 2009;14(4):421-426.

Cooper RG, St Clair Forbes W, Jayson MI. Radiographic demonstration of paraspinal muscle wasting in patients with chronic low back pain. Br J Rheumatol. 1992;31(6):389-394.

Crossley KM, Bennell KL, Cowan SM, et al. Analysis of outcome measures for persons with patellofemoral pain: Which are reliable and valid? Arch Phys Med Rehabil. 2004;85(5):815-822.

Hayes K, Walton JR, Szomor ZL, et al. Reliability of 3 methods for assessing shoulder strength. J 
Shoulder Elbow Surg. 2002;11(1):33-39.

Ishida $\mathrm{H}$, Watanabe $\mathrm{S}$. Influence of inward pressure of the transducer on lateral abdominal muscle thickness during ultrasound imaging. J Orthop Sports Phys Ther. 2012;42(9):815-818.

Jhu JL, Chai HM, Jan MH, et al. Reliability and relationship between 2 measurements of transversus abdominis dimension taken during an abdominal drawing-in maneuver using a novel approach of ultrasound imaging. J Orthop Sports Phys Ther. 2010;40(12):826-832.

Keagy RD, Brumlik J, Bergan JL. Direct electromyography of the psoas major muscle in man. J Bone Joint Surg. 1966;48(7):1377-1382.

Nachemson A. Electromyographic studies on the vertebral portion of the psoas muscle; with special reference to its stabilizing function of the lumbar spine. Acta Ortho Scand. 1966;37(2): 177-190.

Nachemson A. The possible importance of the psoas muscle for stabilization of the lumbar spine. Acta Ortho Scand. 1968;39(1):47-57.

McGill SM, Santaguida L, Stevens J. Measurement of the trunk musculature from T5 to L5 using MRI scans of 15 young males corrected for muscle fibre orientation. Clin Biomech (Bristol, Avon). 1993;8(4):171-178.

Sajko S, Stuber K. Psoas major: A case report and review of its anatomy, biomechanics, and clinical implications. J Can Chiropr Assoc. 2009;53(4): 311-318.

Santaguida PL, McGill SM. The psoas major muscle:
A three-dimensional geometric study. J Biomech. 1995;28(3):339-345.

Sipilä S, Suominen H. Ultrasound imaging of the quadriceps muscle in elderly athletes and untrained men. Muscle Nerve. 1991;14(6):527-533.

Snijder MB, Visser M, Dekker JM, et al. The prediction of visceral fat by dual-energy $\mathrm{x}^{-}$-ray $\mathrm{ab}$ sorptiometry in the elderly: A comparison with computed tomography and anthropometry. Int J Obes Relat Metab Disord. 2002;26(7):984-993.

Takai Y, Katsumata Y, Kawakami Y, et al. Ultrasound method for estimating the cross ${ }^{-} \mathrm{sec}^{-}$ tional area of the psoas major muscle. Med Sci Sports Exerc. 2011;43(10):2000-2004.

Tatu L, Parratte B, Vuillier F, et al. Descriptive anatomy of the femoral portion of the iliopsoas muscle. Anatomical basis of anterior snapping of the hip. Surg Radiol Anat. 2001;23(6):371-374.

Teyhen DS, Gill NW, Whittaker JL, et al. Rehabilitative ultrasound imaging of the abdominal muscles. J Orthop Sports Phys Ther. 2007;37 (8):450-466.

Wallwork TL, Hides JA, Stanton WR. Intrarater and interrater reliability of assessment of lumbar multifidus muscle thickness using rehabilitative ultrasound imaging. J Orthop Sports Phys Ther. 2007;37(10):608-612

This article was received September 24, 2013, was reviewed September 24, 2013, and was accepted October 30, 2013. 